\title{
Theory of the Luminescence Spectra of High-Density Electron-Hole Systems: Crossover from Excitonic Bose-Einstein Condensation to Electron-Hole BCS state
}

\author{
T. J. Inagaki ${ }^{\mathrm{a}, 1}$ M. Aihara ${ }^{\mathrm{a}}$ A. Takahashi ${ }^{\mathrm{a}}$ \\ ${ }^{a}$ Graduate School of Materials Science, Nara Institute of Science and Technology, \\ Ikoma, Nara 630-0101, Japan
}

\begin{abstract}
We present a unified theory of luminescence spectra for highly excited semiconductors, which is applicable both to the electron-hole BCS state and to the exciton Bose-Einstein condensate. The crossover behavior between electron-hole BCS state and exciton Bose-Einstein condensate clearly manifests itself in the calculated luminescence spectra. The analysis is based on the Bethe-Salpeter equation combined with the generalized random-phase-approximation, which enables us to consider the multiple Coulomb scattering and the quantum fluctuation associated with the center-of-mass motion of electron-hole pairs. In the crossover regime, the calculated spectra are essentially different from results obtained by the BCS-like mean-field theory and the interacting Boson model. In particular, it is found that the broad spectrum, arising from the recombination of electron-hole BCS state, splits into the $\mathrm{P}$ - and $\mathrm{P}_{2}$-luminescence bands with decreasing the particle density. The dependence of these bands on the carrier density is in good agreement with experiments for highly excited semiconductors.
\end{abstract}

Key words: A. semiconductors, D. optical properties, D. phase transitions, D. electron-electron interactions

The issue of Bose-Einstein condensation (BEC) of excitons has received considerable attention because of the Bosonic nature of excitons [1]. Following the pioneering work by Keldysh et al. [2], the high-density electron-hole system with excitonic instability has been extensively studied both theoretically [3-5] and experimentally [6]. Recent developments in the experimental techniques make it possible to observe the remarkable optical phenomena suggesting the

$\overline{1}$ E-mail:inagaki@ms.aist-nara.ac.jp

Preprint submitted to Elsevier Preprint 27 October 2018 
spontaneous generation of the macroscopic quantum coherence. In particular, the anomalous exciton transport phenomena observed in $\mathrm{Cu}_{2} \mathrm{O}$ [7] and $\mathrm{BiI}_{3}$ [8] are manifestations of the nondissipative rapid propagation of high-density excitons. However, current understanding of these experiments still remains controversial because of the complicated experimental situation due to the finite life-time of excitons, phonon effects, spatial inhomogeneity of the system, and so forth.

We should remark that the macroscopic quantum state of high-density excitons is qualitatively different from the BEC states of superfluid ${ }^{4} \mathrm{He}$ and atomic gases. The system exhibits a crossover from the exciton BEC to the electron-hole BCS state with increasing the excitation-light intensity.

Under the weak photoexcitation, the system can be described by the interacting exciton model [3] because the mean distance between excitons is much larger than the exciton Bohr radius. The system undergoes the exciton BEC at sufficiently low temperatures, and the density of exciton with zero momentum is the order parameter of the macroscopic quantum state. When the photoexcitation is sufficiently strong, the bound electron-hole pairs can no longer be regarded as pure Bosons because the state-filling and the exchange effects, originated from the Fermionic nature of electrons and holes, take essential part in the problem. In the high-density limit, the electron-hole pairs behave like Cooper pairs, and the BCS-like energy gap at the Fermi level is the order parameter of the macroscopic quantum state $[4,9,10]$ which is essentially the same as the excitonic phase [2]; it is noted that no Bosonic nature exists above $\mathrm{T}_{\mathrm{c}}$, and the property of the system is totally different from the exciton BEC state.

Similar crossover phenomena have been discussed in a variety of physical contexts, e.g., superconductivity [11], nuclear matter [12] and superfluid ${ }^{3} \mathrm{He}$ [13]. Recently, much attention has been focused on the BCS-BEC crossover in connection with the unusual properties of the high- $T_{\mathrm{c}}$ cuprate superconductors. In particular, it is observed that a pseudo-gap structure in the normal-state density of states of underdoped cuprates persists almost up to room temperature [14]. A further unusual property of the high- $T_{\mathrm{c}}$ superconductor is its extreme short coherence length (of the order of several lattice constants) of Cooper pairs $[15,16]$; this fact is in contrast with the conventional superconductors where the Cooper pairs are strongly overlapping in real space. When we investigate this kind of crossover phenomena, the optically generated electron-hole system has the marked advantage over the superconducting materials, because the macroscopic quantum states can directly be controlled by changing the intensity or the frequency of excitation light.

In this article, we first present a unified theory of luminescence spectra for highly excited electron-hole systems which is applicable both to the electron- 
hole BCS state and to the exciton BEC state. The earlier theories based on the interacting-Boson model or on the BCS-like mean-field theory are not appropriate because the highly photoexcited system is often in the intermediate state between the electron-hole BCS state and the exciton BEC state. In the present theory, the multiple Coulomb interaction between electron-hole pairs is incorporated by numerically solving the Bethe-Salpeter (BS) equation [17] for electron-hole pair correlation function. This analysis is closely related to that in Ref. [18], where the absorption spectra for condensed exciton system are calculated by properly taking into account the ladder diagram for vertex function. In the present paper, the effect of the collective quantum fluctuation associated with the center-of-mass motion of electron-hole pairs is incorporated by the generalized random-phase approximation (RPA) [19]. The calculated spectra considerably deviate from results obtained by the BCS-like mean-field analysis, and are essentially different from results by the interacting Boson model. The crossover from the exciton BEC to the electron-hole BCS state is clearly found to manifest itself in the luminescence spectra. In particular, the $\mathrm{P}_{2^{-}}$ luminescence band, which arises from the exciton recombination accompanied by the excitation of another exciton from $1 \mathrm{~S}$ to $2 \mathrm{~S}$ state, continuously changes to the Fermi-edge singularity with increasing the electron-hole density. In the low carrier density, we find that the intensity of the $\mathrm{P}_{2}$-luminescence band predominates comparing to that of the P-luminescence band, and this behavior is in excellent agreement with experiments for various semiconductors $[20,21]$. The present analysis also predicts the weak luminescence band due to the recombination of electron-hole pairs which are generated by the collective phase fluctuation. These result are closely related to the recent noteworthy experiment of $\mathrm{ZnO}$ thin films, in which the room temperature ultraviolet laser emission is observed [22].

We consider a electron-hole system in a direct-gap semiconductor, which consists of the isotropic, nondegenerate parabolic conduction and valence bands with identical electron and hole masses. The repulsive interactions between electrons and between holes as well as the electron-hole attractive interaction are taken into account. The spin degrees of freedom is neglected to avoid the unnecessary complication due to the biexciton state.

The spontaneous emission rate is expressed by the imaginary part of the electron-hole pair correlation function as $I(\omega)=-2 \operatorname{Im} G(\omega-\mu+i \gamma)$, where $\gamma$ and $\mu$ are the exciton decay constant and the chemical potential of the electron-hole pairs, respectively. $G(\omega)$ is the Fourier transform of the electronhole pair correlation function

$$
G(t)=-i \Theta(t) \sum_{\boldsymbol{k}, \boldsymbol{p}} g_{\boldsymbol{k}} g_{\boldsymbol{p}}\left\langle c_{\boldsymbol{k}}^{\dagger}(0) d_{-\boldsymbol{k}}^{\dagger}(0) d_{-\boldsymbol{p}}(t) c_{\boldsymbol{p}}(t)\right\rangle
$$

where $g_{\boldsymbol{k}}$ is the radiation-matter coupling constant; $c_{\boldsymbol{p}}$ and $d_{\boldsymbol{p}}$ are the anni- 
hilation operators for electrons and holes, respectively. Here $\langle\cdots\rangle$ indicates the expectation value with respect to the electron-hole BCS state. In order to consider the electron-hole pair correlation, let us introduce the Bogoliubov transformation given by $c_{\boldsymbol{k}}=u_{\boldsymbol{k}} \alpha_{\boldsymbol{k}}+v_{\boldsymbol{k}} \beta_{-\boldsymbol{k}}^{\dagger}, d_{-\boldsymbol{k}}=u_{\boldsymbol{k}} \beta_{-\boldsymbol{k}}-v_{\boldsymbol{k}} \alpha_{\boldsymbol{k}}^{\dagger}$, where $\alpha_{\boldsymbol{k}}$ and $\beta_{-\boldsymbol{k}}$ are the annihilation operators for new quasiparticles (Bogolons). The Bogoliubov parameters $u_{\boldsymbol{k}}$ and $v_{\boldsymbol{k}}$ are determined by solving the BCS-like gap equation for electron-hole systems. The BCS-like gap equation is reduced to the Wannier equation in the low density limit, which implies that the equation gives the correct result for the relative motion of electron-hole pairs both in the high- and low-density limits [11].

In the following analysis, it is convenient to introduce a two-component operator, $\psi_{\boldsymbol{k}}=\left(\alpha_{\boldsymbol{k}}, \beta_{-\boldsymbol{k}}^{\dagger}\right)$ and the Pauli spin matrices $\boldsymbol{\tau}_{\mu}(\mu=1,2,3)$. The spontaneous emission rate is expressed as

$$
I(\omega)=\frac{2 \gamma I_{\mathrm{coh}}}{(\omega-\mu)^{2}+\gamma^{2}}-\frac{1}{4} \operatorname{Im}\left[\sum_{\boldsymbol{k}, \boldsymbol{p}} g_{\boldsymbol{k}} g_{\boldsymbol{p}} \boldsymbol{K}_{\boldsymbol{k}} \mathcal{G}_{\boldsymbol{k}, \boldsymbol{p}}(\omega-\mu+i \gamma) \boldsymbol{K}_{\boldsymbol{p}}\right]
$$

where $\boldsymbol{K}_{\boldsymbol{k}}=\left(u_{\boldsymbol{k}}^{2}-v_{\boldsymbol{k}}^{2}, i\right)$. Here $I_{\text {coh }}$ is defined by

$$
I_{\mathrm{coh}}=\sum_{\boldsymbol{k}, \boldsymbol{p}} g_{\boldsymbol{k}} g_{\boldsymbol{p}} u_{\boldsymbol{k}} v_{\boldsymbol{k}} u_{\boldsymbol{p}} v_{\boldsymbol{p}}\left\langle\left(\psi_{\boldsymbol{k}}^{\dagger} \boldsymbol{\tau}_{3} \psi_{\boldsymbol{k}}\right)\left(\psi_{\boldsymbol{p}}^{\dagger} \boldsymbol{\tau}_{3} \psi_{\boldsymbol{p}}\right)\right\rangle
$$

and the $(\mu, \nu)$ component of $\mathcal{G}_{\boldsymbol{k}, \boldsymbol{p}}(\omega)$ is defined by the Fourier transform of

$$
\left(\mathcal{G}_{\boldsymbol{k}, \boldsymbol{p}}(t)\right)_{\mu, \nu}=-i \Theta(t)\left\langle\left(\psi_{\boldsymbol{k}}^{\dagger}(0) \boldsymbol{\tau}_{\mu} \psi_{\boldsymbol{k}}(0)\right)\left(\psi_{\boldsymbol{p}}^{\dagger}(t) \boldsymbol{\tau}_{\nu} \psi_{\boldsymbol{p}}(t)\right)\right\rangle
$$

respectively. The first term on the right-hand side of Eq. (2) gives the the sharp spectrum at the chemical potential of the electron-hole pairs; this spectral component arises from the coherent spontaneous emission from the macroscopic quantum state. The electron-hole pair recombination does not generate Bogolons so that the spectral width is merely determined by the lifetime of electrons in the conduction band. The second term expresses the incoherent spontaneous emission rate accompanied by the creation of Bogolons; this spectral component reflects the various interesting features arising from the many-body interaction in high-density electron-hole systems.

Making use of the generalized RPA, we evaluate $I_{\text {coh }}$ in Eq. (2) as

$$
I_{\mathrm{coh}}=\left\{\sum_{\boldsymbol{k}} g_{\boldsymbol{k}} u_{\boldsymbol{k}} v_{\boldsymbol{k}}\right\}^{2}+2 \sum_{\boldsymbol{k}, \boldsymbol{p}} g_{\boldsymbol{k}} g_{\boldsymbol{p}} u_{\boldsymbol{k}} v_{\boldsymbol{k}} u_{\boldsymbol{p}} v_{\boldsymbol{p}} \int_{-\infty}^{+\infty} \frac{d z}{2 \pi \mathrm{i}} \frac{V_{\boldsymbol{k}-\boldsymbol{p}}(z)}{\left(z-E_{\boldsymbol{k}}-E_{\boldsymbol{p}}\right)^{2}}
$$


where $V_{\boldsymbol{q}}(\omega)$ is the screened Coulomb potential. The single particle excitation energy (Bogolon energy) is given by $E_{\boldsymbol{k}}=\sqrt{\zeta_{\boldsymbol{k}}^{2}+\Delta_{\boldsymbol{k}}^{2}}$, where $\zeta_{\boldsymbol{k}}$ and $\Delta_{\boldsymbol{k}}$ are the renormalized energy of the electron-hole pair and the BCS gap, respectively. The first term on the right-hand side of Eq. (5) is obtained by the BCS-like mean field approximation, and the second term expresses the correction which comes from the collective quantum fluctuations similar to the Anderson mode in superconductivity [19].

The function $\mathcal{G}_{\boldsymbol{k}, \boldsymbol{p}}(\omega)$, which gives the intensity of the incoherent emission through Eq. (2), satisfies the following BS equation,

$$
\begin{array}{r}
\mathcal{G}_{\boldsymbol{p}, \boldsymbol{k}}(\omega)\left(\omega \boldsymbol{\tau}_{0}+2 E_{\boldsymbol{k}} \boldsymbol{\tau}_{2}\right)-\sum_{\boldsymbol{k}^{\prime}} V_{\boldsymbol{k}-\boldsymbol{k}^{\prime}}(\omega) \mathcal{G}_{\boldsymbol{p}, \boldsymbol{k}^{\prime}}(\omega)\left(C_{\boldsymbol{k}, \boldsymbol{k}^{\prime}}^{(0)} \boldsymbol{\tau}_{2}+i C_{\boldsymbol{k}, \boldsymbol{k}^{\prime}}^{(3)} \boldsymbol{\tau}_{1}\right) \\
=\left\langle\left(\psi_{\boldsymbol{k}}^{\dagger}(0) \boldsymbol{\tau}_{\mu} \psi_{\boldsymbol{k}}(0)\right)\left(\psi_{\boldsymbol{p}}^{\dagger}(0) \boldsymbol{\tau}_{\nu} \psi_{\boldsymbol{p}}(0)\right)\right\rangle
\end{array}
$$

where $C_{\boldsymbol{k}, \boldsymbol{p}}^{(0)} \equiv\left(u_{\boldsymbol{k}} u_{\boldsymbol{p}}+v_{\boldsymbol{k}} v_{\boldsymbol{p}}\right)^{2}$ and $C_{\boldsymbol{k}, \boldsymbol{p}}^{(3)} \equiv\left(u_{\boldsymbol{k}} v_{\boldsymbol{p}}-v_{\boldsymbol{k}} u_{\boldsymbol{p}}\right)^{2}$ are the coherence factors and $\boldsymbol{\tau}_{0}$ is the $2 \times 2$ unit matrix. This BS equation is obtained by linearizing the equation-of-motion for the correlation function $\mathcal{G}_{\boldsymbol{k}, \boldsymbol{p}}(t)$. The multiple Coulomb interaction between electrons and holes is expressed by the second term on the right-hand side of Eq. (6). This term makes a significant contribution to the recombination of the electron-hole pairs with nonzero total momentum, which is not taken into account in the BCS-like mean-field theory. The right-hand side of Eq. (6) is rewritten as

$$
\begin{array}{r}
\left\langle\left(\psi_{\boldsymbol{k}}^{\dagger}(0) \boldsymbol{\tau}_{\mu} \psi_{\boldsymbol{k}}(0)\right)\left(\psi_{\boldsymbol{p}}^{\dagger}(0) \boldsymbol{\tau}_{\nu} \psi_{\boldsymbol{p}}(0)\right)\right\rangle \\
=\left(\boldsymbol{\tau}_{0}+\boldsymbol{\tau}_{2}\right) \delta_{\boldsymbol{k}, \boldsymbol{p}}-\frac{1}{2} \boldsymbol{\tau}_{3} \sum_{j=1}^{2}(-1)^{j+1}\left\langle\left(\psi_{\boldsymbol{k}}^{\dagger}(0) \boldsymbol{\tau}_{j} \psi_{\boldsymbol{p}}(0)\right)\left(\psi_{\boldsymbol{k}}^{\dagger}(0) \boldsymbol{\tau}_{j} \psi_{\boldsymbol{p}}(0)\right)\right\rangle
\end{array}
$$

The second term on the right-hand side represents the fluctuation effect due to the collective excitations similar to the Anderson mode in superconductivity. A generalized RPA analysis gives the following expression,

$$
\sum_{j=1}^{2}(-1)^{j+1}\left\langle\left(\psi_{\boldsymbol{k}}^{\dagger}(0) \boldsymbol{\tau}_{j} \psi_{\boldsymbol{p}}(0)\right)\left(\psi_{\boldsymbol{k}}^{\dagger}(0) \boldsymbol{\tau}_{j} \psi_{\boldsymbol{p}}(0)\right)\right\rangle=4 \int_{-\infty}^{+\infty} \frac{d z}{2 \pi i} \frac{V_{\boldsymbol{p}-\boldsymbol{k}}(z) C_{\boldsymbol{p}, \boldsymbol{k}}^{(3)}}{z^{2}-\left(E_{\boldsymbol{p}}+E_{\boldsymbol{k}}\right)^{2}}
$$

As a first step in our numerical calculations, the gap equation is iteratively solved to evaluate the renormalized energy of electron-hole pair $\zeta_{\boldsymbol{k}}$ and the BCS gap function $\Delta_{\boldsymbol{k}}$. The incoherent luminescence spectrum is evaluated by numerically calculating the eigenvalues and eigenvectors of the stability 
matrix [23] for Eq. (6). The quasi-static dielectric function given by the singleplasmon-pole approximation is employed $[5,9]$.

The incoherent components of the luminescence spectra are shown in Figs. 13. As a reference, we also present the luminescence spectra calculated by the BCS-like mean-field theory and the generalized RPA analysis, in which the fluctuation effect associated with the center-of-mass motion of pairs is considered but the multiple Coulomb interaction is neglected. We use the units where the exciton binding energy and the exciton Bohr radius being unity. The dimensionless mean interparticle distance $r_{\mathrm{s}}=(4 \pi n / 3)^{-1 / 3}$ is introduced, where $n$ is the electron-hole density. The exciton decay constant is chosen as $\gamma=0.03$, unless othewise stated. The arrow in the figures represents the level of the chemical potential of the electron-hole pair at which the coherent luminescence line appears. With decreasing the particle density, the position of coherent luminescence band approaches to $\omega-E_{\mathrm{g}}=-1$, where $E_{\mathrm{g}}$ is the energy gap between the valence and conduction bands. This behavior ensures that the present analysis reproduces the result obtained by the interacting exciton model in the low density limit.

Fig. 1 shows the luminescence spectrum in the high density case $\left(r_{\mathrm{s}}=1.0\right)$. The band renormalization effect due to the exchange interaction is clearly observed. The peaked structure near the Fermi level $\left(\omega-E_{\mathrm{g}} \simeq 1\right)$ is the manifestation of the BCS-like gap formation, which is strongly pronounced by the multiple Coulomb interaction between electrons and holes; the electron-hole pairs with nonzero total momentum take part in the recombination processes. The luminescence band in $\omega-E_{\mathrm{g}}>\mu$, which is missing in the mean-field analysis, corresponds to the pair annihilation process of the virtual Bogolons generated by the collective phase fluctuation.

The luminescence spectra in the intermediate densities corresponding to $r_{\mathrm{s}}=$ 2.2 is shown in Fig. 2. It is noted that the incoherent luminescence spectrum below the chemical potential splits into two components: the broad component below $\omega-E_{\mathrm{g}} \simeq-1.7$ and the sharp one around $\omega-E_{\mathrm{g}} \simeq-1.5$. The intensity of the broad component is approximately proportional to the square of the particle density; its spectral position slightly shifts towards the shorter wavelength with decreasing the particle density. These behaviors suggest that the broad component corresponds to the P-luminescence band [20,21,24], which comes from the radiative recombination of an exciton assisted by the dissociation of another exciton. On the other hand, the sharp component around $\omega-E_{\mathrm{g}} \simeq-1.5$ corresponds to the $\mathrm{P}_{2}$-luminescence band [20,21], which arises from the radiative recombination of an exciton accompanied by the excitation of another exciton from $1 \mathrm{~S}$ to $2 \mathrm{~S}$ state. In addition, a weak luminescence subband, called $\mathrm{P}_{3}$, is observed around $\omega-E_{\mathrm{g}} \simeq-1.6$; this component comes from the exciton recombination assisted by the excitation of another exciton from $1 \mathrm{~S}$ to $3 \mathrm{~S}$ state. The peaked structure above the chemical potential originates 
from the pair annihilation of Bogolons, which are generated by the collective phase fluctuation from the electron-hole BCS state. It is noted that we do not deal with a simple two-exciton problem, but calculated $\mathrm{P}, \mathrm{P}_{2}$, and $\mathrm{P}_{3}$ bands in the framework of the many-body theory. We should also recall that these spectral components cannot be obtained by a BCS-like mean-field theory with the RPA correction.

The luminescence spectrum in a low electron-hole density state $\left(r_{\mathrm{s}}=13\right)$ is shown in Fig. 3. It is found that the P-luminescence band almost disappears and the $\mathrm{P}_{2}$ band predominates with decreasing the carrier density. This behavior arises because the transition of another exciton to the bound state $(2 \mathrm{~S})$ is much stronger than that to the dissociated states in low carrier density state.

We show in Fig, 4 the comparison between the present theory and the experiment for $\mathrm{ZnO}$ thin film at 77K [20]. Here the exciton decay constant is chosen as $\gamma=0.1$. The calculated spectra are in good agreements with this experimant in the following points: (1) the exciton luminescence line predominates in the low density state. (2) The intensity of the $\mathrm{P}_{2}$-luminescence line grows superlinearly with increasing carrier density, and it prevails in the intermediate density states. (3) The P-luminescence line appears in higher densities and it merges with the $\mathrm{P}_{2}$-luminescence line as the carrier density increases.

Finally, it should be noted that the dependence of the line shape and the spectral position on the electron-hole density cannot be predicted by the wellknown analysis for two-exciton problem [25].

In conclusion, we first present the many-body theory for luminescence spectra which is applicable both to the exciton BEC state and to the electron-hole BCS state. Our analysis, based on the BS equation combined with the generalized RPA, properly describes the crossover behavior between the two essentially different macroscopic quantum states.

This work is partially supported by a Grant-in-Aid for Scientific Research from the Ministry of Education, Science, Culture and Sports of Japan.

\section{References}

[1] S. A. Moskalenko, Fiz. Tverd. Tela 4, (1962) 276; I. M. Blatt, K. W. Boer and W. Brandt, Phys. Rev. 126, (1962) 1691.

[2] L. V. Keldysh and Yu. V. Kopaev, Sov. Phys. Solid State 6, (1965) 2219; L. V. Keldysh and A. N. Kozlov, Sov. Phys. JETP 27, (1968) 521; R. R. Guseinov and L. V. Keldysh, Sov. Phys. JETP 36, (1973) 1193.

[3] E. Hanamura and H. Haug, Phys. Rep. 33, (1977) 209; 
[4] C. Comte and P. Nozières, J. Phys. (Paris) 43, (1982) 1069; P. Nozières and C. Comte, J. Phys. (Paris) 43, (1982) 1083; L. V. Keldysh, in Bose-Einstein Condensation, edited by A. Griffin, D. W. Snoke and S. Stringari, Cambridge University Press, Cambridge, 1995, p. 246.

[5] H. Haug and S. Schmitt-Rink, Prog. Quantum Electron. 9, (1988) 3.

[6] D. Hulin, A. Mysyrowicz and C. Benoit à la Guillaume, Phys. Rev. Lett. 45, (1980) 1970; D. W. Snoke, J. P. Wolfe and A. Mysyrowicz, Phys. Rev. B 41, (1990) 11171; J. L. Lin and J. P. Wolfe, Phys. Rev. Lett. 71, (1993) 1222; T. Goto, M. Y. Shen, S. Koyama and T. Yokouchi, Phys. Rev. B 55, (1997) 7609.

[7] E. Fortin, S. Farad and A. Mysyrowicz, Phys. Rev. Lett. 70, (1993) 3951; A. Mysyrowicz, E. Benson and E. Fortin, Phys. Rev. Lett. 77, (1996) 896.

[8] H. Kondo, T. Kawai, T. Karasawa, I. Akai and T. Komatsu, J. Luminescence 66 \& 67, (1996) 448; H. Kondo, H. Mino, I. Akai and T. Karasawa, Phys. Rev. B 58, (1998) 13835.

[9] R. Zimmermann, Phys. Stat. Sol. B 76, (1976) 191.

[10] M. Lindberg and S. W. Koch, Phys. Rev. B 38, (1988) 3342.

[11] P. Nozières and S. Schmitt-Rink, J. Low Temp. Phys. 59, (1985) 195.

[12] Y. Wada, Prog. Theor. Phys. 24 (1960) 920; 25713 (1961); 26 (1961) 321.

[13] A. J. Leggett, in Modern Trends in the Theory of Condensed Matter, edited by A. Pȩkalski and J. Przystawa Springer-Verlag, Berlin 1980, p. 14.

[14] H. Ding et al., Nature 382, (1996) 51; A. G. Loeser et al., Science 273, (1996) 325; P. Coleman, Nature 392, (1998) 134; Ch. Renner et al., Phys. Rev. Lett. 80, (1998) 149.

[15] Y. J. Uemura et al., Phys. Rev. Lett. 66, (1991) 2665; Nature 352, (1991) 605.

[16] For a review on the theory of the crossover from BEC to BCS, see, e.g., M. Randeria, in Bose-Einstein Condensation, edited by A. Griffin, D. W. Snoke and S. Stringari, Cambridge University Press, Cambridge, 1995, p. 355.

[17] See, e.g., N. Nakanishi, Prog. Theor. Phys. Suppl. (Kyoto) 43, (1969) 1; 95, (1988) 1.

[18] H. Chu and Y. C. Chang, Phys. Rev. B 54, (1996) 5020.

[19] P. W. Anderson, Phys. Rev. 112, (1958) 1900; G. Rickayzen, Phys. Rev. 115, (1959) 795; A. Bardasis and J. R. Schrieffer, Phys. Rev. 121, (1961) 1050.

[20] H. J. Ko et al., Appl. Phys. Lett. 76, (2000) 1905.

[21] J. M. Hvam, Phys. Stat. Sol. B 63, (1974) 511; P. Yu, et al., in Proceedings of the 23rd International Conference on the Physics of Semiconductors, edited by M. Scheffler and R. Zimmermann World Scientific, Singapore, 1996, Vol. 2, p. 1453; H. Liebing, P. Mengel and W. Ruppel, Phys. Stat. Sol. B 72, (1975) 
431; J. M. Hvam and E. Ejder, J. Luminescence 12\&13, (1976) 611; A. Mercier and J. P. Voitchovsky, Phys. Rev. B 11, (1975) 2243; A. Opanowicz et al., Phys. Stat. Sol. B 75, (1976) 471; W. Maier and C. Klingshirn, Solid State Commun. 28, (1978) 13.

[22] Z. K, Tang et al., Appl. Phys. Lett. 72, (1998) 3270.

[23] P. Ring and P. Schuck, The Nuclear Many-Body Problem, Springer, New York, 1980.

[24] C. Benoit à la Guillaume, J. -M. Debever and F. Salvan, Phys. Rev. 177, (1969) 567; J. Bille, H. Liebing and P. Mengel, Phys. Stat. Sol. B 53, (1972) 353.

[25] T. Moriya and T. Kushida, J. Phys. Soc. Jpn. 40 (1976) 1668; 40 (1976) 1676; T. Moriya and T. Kushida, J. Luminescence 12\&13 (9176) 617; H. Büttner, Phys. Stat. Sol. 42 (1970) 775.

Fig. 1. The luminescence spectrum in the high-density state $\left(r_{\mathrm{s}}=1.0\right)$ calculated by (a) the BCS-like mean-field theory, (b) the generalized RPA, and (c) the present theory.

Fig. 2. The luminescence spectrum in the intermediate-density state $\left(r_{\mathrm{s}}=2.2\right)$ calculated by (a) the BCS-like mean-field theory, (b) the generalized RPA, and (c) the present theory.

Fig. 3. The luminescence spectrum in the low-density state $\left(r_{\mathrm{s}}=13\right)$ calculated by (a) the BCS-like mean-field theory, (b) the generalized RPA, and (c) the present theory.

Fig. 4. The luminescence spectrum for various density states: (a) the present theory, (b) experiment for $\mathrm{ZnO}$ thin film at 77K (from Ref. [20]). Here Ex-line corresponds to the free exciton emission. 


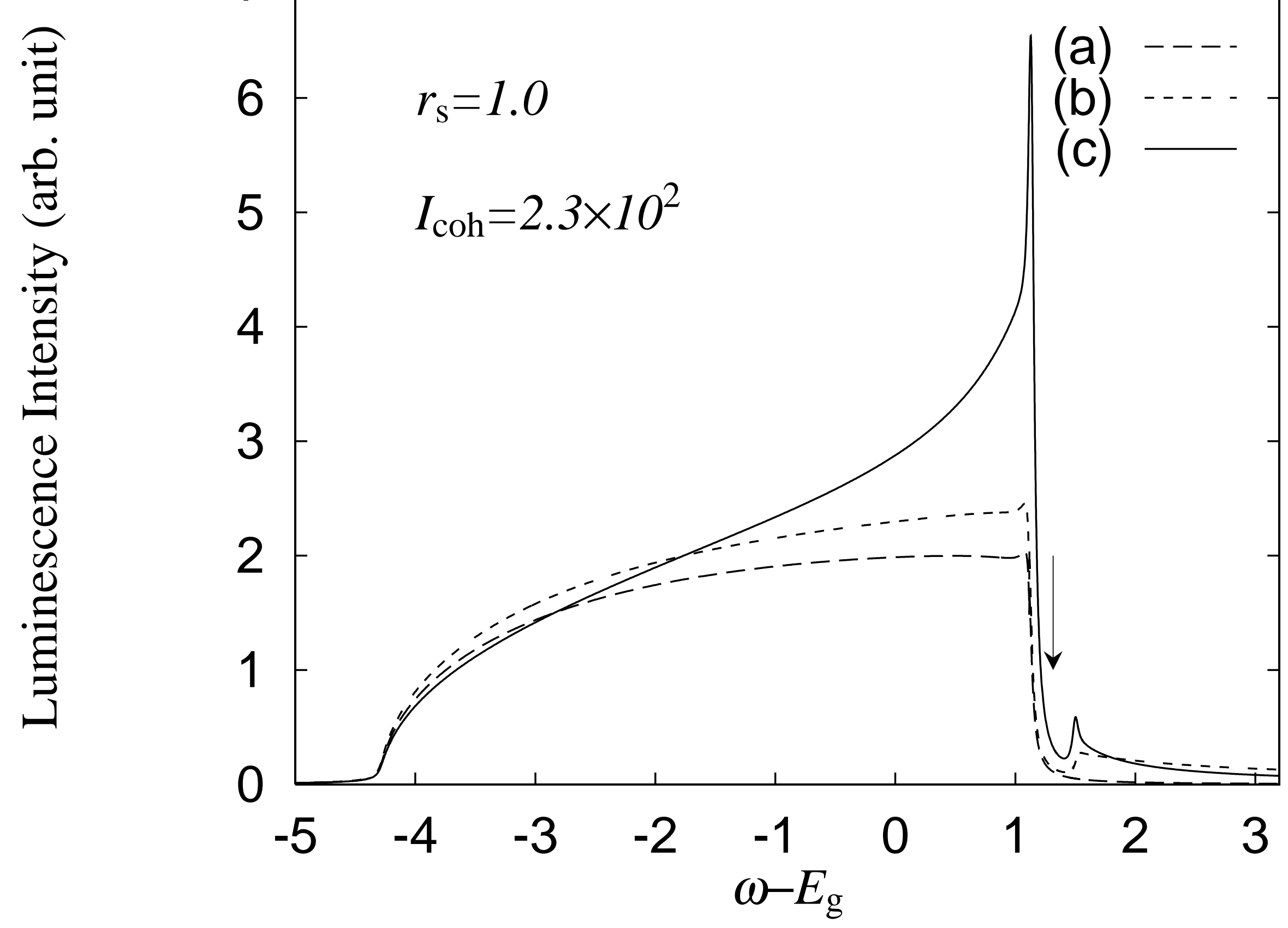




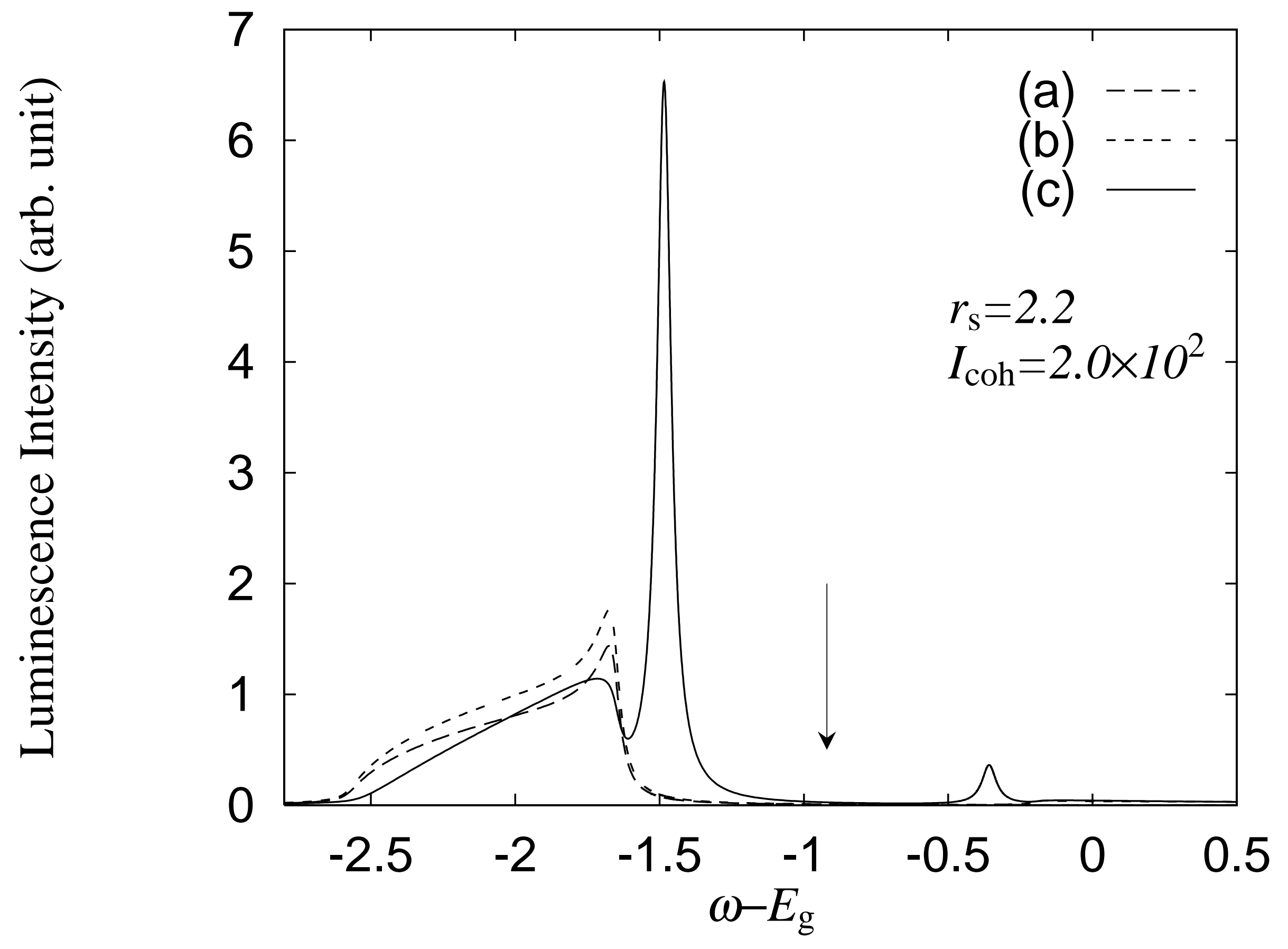




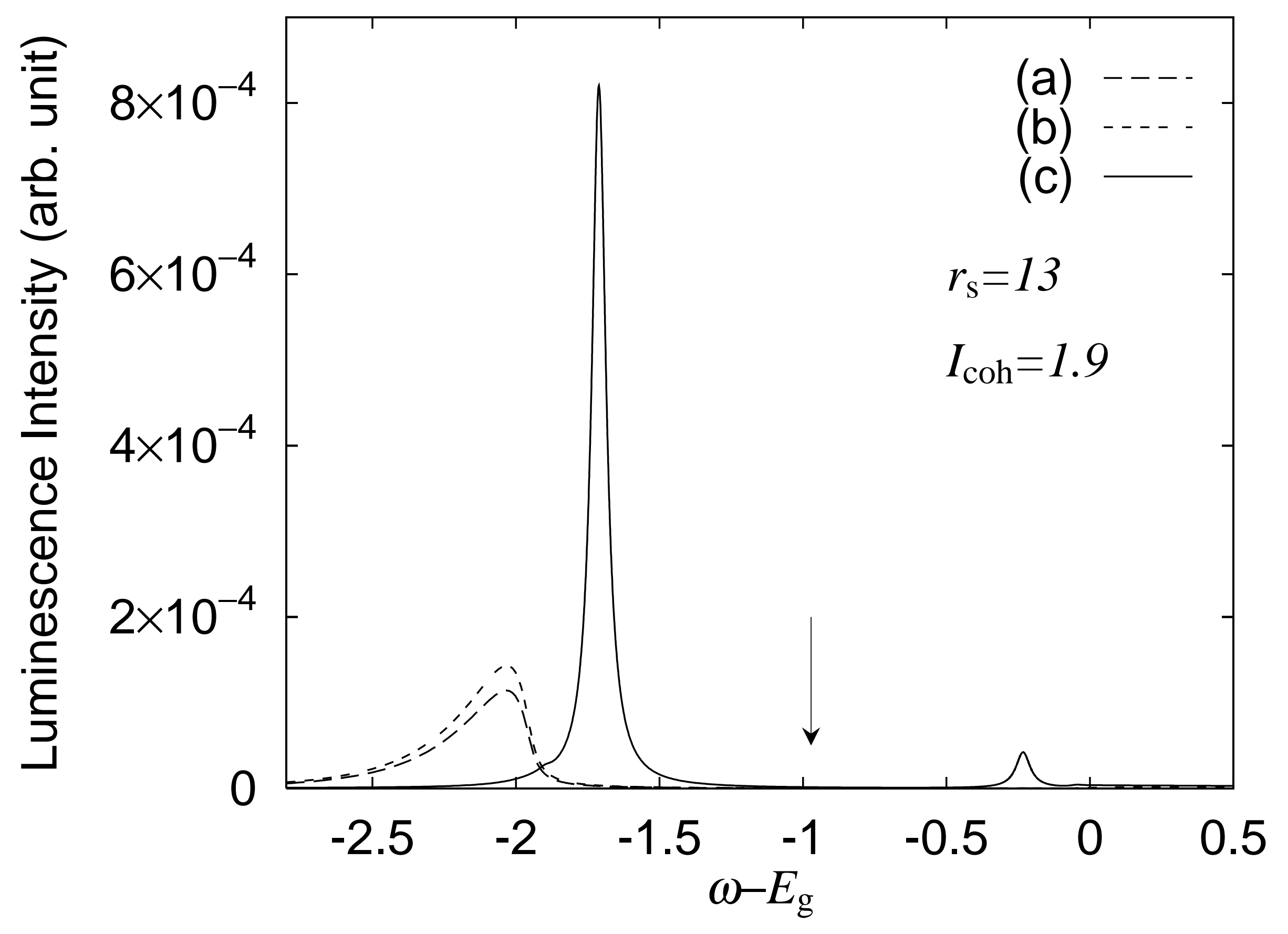

